\title{
Comparison of cardiovascular magnetic resonance with real-time three-dimensional echocardiography and the right ventricular automated systolic index in the assessment of the right ventricular function
}

\author{
Florian Andre ${ }^{1 *}$, Sebastian Greiner ${ }^{1}$, Cihan Celik', Mohamed A Abdelrazek², Maria Fernanda Braggion Santos ${ }^{3}$, \\ Dirk Lossnitzer ${ }^{1}$
}

From 16th Annual SCMR Scientific Sessions

San Francisco, CA, USA. 31 January - 3 February 2013

\section{Background}

The right ventricular (RV) function has an important diagnostic value in many cardiopulmonary diseases and is a predictor for the long-term outcome. Cardiovascular magnetic resonance (CMR) is the gold-standard for the $\mathrm{RV}$ quantification as the complex anatomy of the RV, i.e. its crescentic shape, impedes reliable measurement by two-dimensional echocardiography (EC). Yet, CMR or real-time 3D echocardiography (RT3DE) measurements are time consuming. Recently, a novel EC parameter, the RV automated systolic index (RV-ASI), has been introduced which employs semi-automated whole-cycle endocardial border detection and calculates volume changes based on the sum-of-discs method (modified Simpson's rule). In this study we evaluate the measurement agreements of two novel EC parameters, a) the RV-ASI and b) the RT3DE, with the reference standard CMR.

\section{Methods}

We studied 25 patients with different cardiopulmonary diseases (coronary artery disease with preserved left ventricular function $(\mathrm{n}=4)$, ischemic cardiomyopathy $(\mathrm{n}=4)$, dilated cardiomyopathy $(\mathrm{n}=10)$, hypertrophic cardiomyopathy $(\mathrm{n}=1)$, cardiac amyloidosis $(\mathrm{n}=2)$, pulmonary hypertension $(\mathrm{n}=4))$ and 15 healthy subjects. CMR imaging was performed on a $1.5 \mathrm{~T}$ whole-body MRI-scanner applying a cine SSFP sequence with parallel imaging. EC was

'Department of Cardiology, University of Heidelberg, Heidelberg, Germany Full list of author information is available at the end of the article performed within 30 min with a commercially available ultrasound machine (GE Vivid E9) including RT3DE and the measurement of the RV-ASI. Student's t-test or Mann-Whitney-Wilcoxon-test respectively as well as a regression analysis and a Bland-Altman-plot were performed. Receiver operator characteristics were calculated for RV-ASI compared to CMR RV ejection fraction (RV$\mathrm{EF})$. A $\mathrm{p}<0.05$ was regarded as statistically significant.

\section{Results}

The RV measurements could be assessed in $100 \%$ of subjects by CMR. RV-ASI was evaluable in 38 of 40 subjects ( $95 \%$ ) by EC. Mean RV-EF measured by CMR was $48 \pm 9 \%$ compared to $51 \pm 10 \%$ by RT3DE and a RVASI of $52 \pm 11 \%$. The correlation between CMR RV-EF and RV-ASI $(\mathrm{r}=0.74, \mathrm{p}<0.0001)$ as well as between CMR RV-EF and RT3DE RV-EF $(r=0.55, p=0.0003)$ were highly significant. The limits of agreement were $\pm 15.1 \%$ for RV-ASI and $\pm 17.4 \%$ for RT3DE RV-EF compared to CMR RV-EF. An RV-ASI cut-off value of $52 \%$ could differentiate between normal and impaired RV function (AUC $=0.92$, sensitivity $=87 \%$, specificity $=93 \%)$ in this mixed study population.

\section{Conclusions}

In this study the time-saving RV-ASI method showed good agreement with CMR regarding the quantification of the RV function. Although it does not provide the measurement of absolute systolic and diastolic RV 
Table 1 Comparison of RT3DE and RV-ASI to CMR

\begin{tabular}{ccccc}
\hline & $r$ & Bias & Limits of agreement & $p$ \\
\hline RT3DE RV-EF (\%) & 0.55 & $+3.1 \%$ & $\pm 17.4 \%$ & 0.0003 \\
\hline RV-ASI (\%) & 0.74 & $+4.4 \%$ & $\pm 15.1 \%$ & $<0.0001$ \\
\hline
\end{tabular}
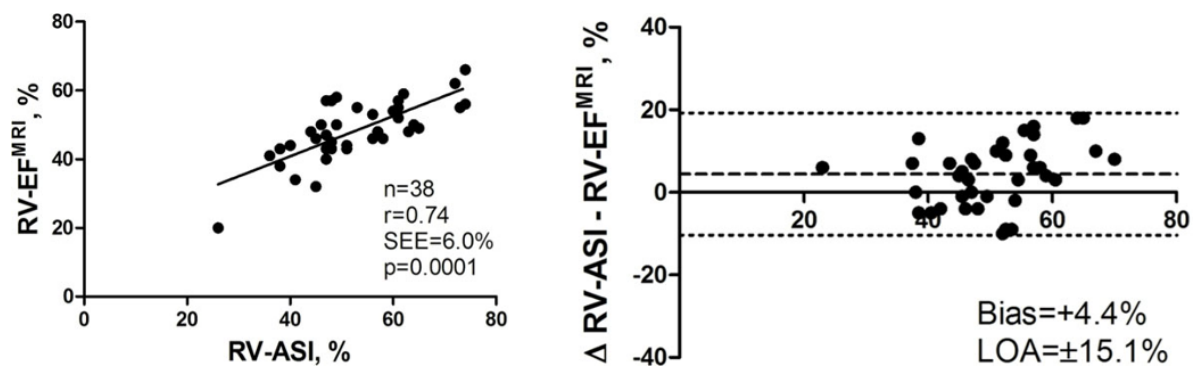

Figure 1 Left: linear regression analysis of RV-ASI and CMR RV-EF. Right: corresponding Bland-Altman-plot of RV-ASI and CMR RV-EF.

volumes, the RV function can be assessed reliably. As it can be obtained easily, it may be utilised for non-invasive follow-up examinations of patients with cardiopulmonary diseases.

\section{Funding}

none

\section{Author details}

${ }^{1}$ Department of Cardiology, University of Heidelberg, Heidelberg, Germany. ${ }^{2}$ Radiology, Cairo university, Faculty of Medicine, Cairo, Egypt. ${ }^{3}$ School of

Medicine of Ribeirao Preto University of Sao Paulo, Sao Paulo, Brazil.

Published: 30 January 2013

doi:10.1186/1532-429X-15-S1-E73

Cite this article as: Andre et al: Comparison of cardiovascular magnetic resonance with real-time three-dimensional echocardiography and the right ventricular automated systolic index in the assessment of the right ventricular function. Journal of Cardiovascular Magnetic Resonance 2013 15(Suppl 1):E73.
Submit your next manuscript to BioMed Central and take full advantage of:

- Convenient online submission

- Thorough peer review

- No space constraints or color figure charges

- Immediate publication on acceptance

- Inclusion in PubMed, CAS, Scopus and Google Scholar

- Research which is freely available for redistribution 